\title{
MicroRNA-155 contributes to the occurrence of epilepsy through the PI3K/Akt/mTOR signaling pathway
}

\author{
WEI DUAN $^{1 *}$, YAN CHEN $^{2 *}$ and XIAO-RONG WANG ${ }^{3 *}$ \\ ${ }^{1}$ Department of Neurology, The Fifth Affiliated Hospital, Harbin Medical University, Harbin, Heilongjiang 150001; \\ ${ }^{2}$ Department of Neurophysiology, Oilfields General Hospital in Daqing, Daqing, Heilongjiang 163001; \\ ${ }^{3}$ Department of Neurology, Ya'an Hospital, Ya'an, Sichuan 625000, P.R. China
}

Received September 5, 2016; Accepted March 15, 2018

DOI: 10.3892/ijmm.2018.3711

\begin{abstract}
Reverse transcription-quantitative polymerase chain reaction (RT-qPCR) was used to measure the expression of microRNA-155 in patients with temporal lobe epilepsy. Commercial kit and western blot analysis were used to measure gap-associated protein expression. The aim of the present study was to investigate the effect of microRNA-155 (miRNA-155) in the occurrence of epilepsy and the molecular mechanism involved. In patients with temporal lobe epilepsy, miRNA-155 expression was evidently higher than that in patients of the normal volunteers group. Overexpression of miRNA-155 resulted in decreased brain-derived neurotrophic factor (BDNF) and tropomyosin receptor kinase B (TrkB) protein expression, increased caspase-3 activity, tumor protein p53 (p53) and apoptosis regulator BAX (Bax) protein expression, and inhibited phosphoinositide 3-kinase (PI3K), phosphorylated (p-)protein kinase B (Akt) and p-mechanistic target of rapamycin (mTOR) protein expression in epilepsy cells. PI3K inhibitor accelerated the effect of miRNA-155 on the inhibition of BDNF and TrkB protein expression, the promotion of caspase- 3 activity, p53 and Bax protein expression, and the inhibition of PI3K, p-Akt and p-mTOR protein expression in epilepsy cells. The present findings indicate that miRNA-155 contributes to the occurrence of epilepsy through the PI3K/Akt/mTOR signaling pathway.
\end{abstract}

\section{Introduction}

Epilepsy is a syndrome caused by the paradoxical discharge of the high synchronization of brain cells, which is caused by either a known or unknown pathogenesis. The clinical manifestation has certain features of a malfunction in the central nervous system, with repeatability, paroxysms, transience and

Correspondence to: Dr Xiao-Rong Wang, Department of Neurology, Ya'an Hospital, 358 Chenghou Road, Ya'an, Sichuan 625000, P.R. China E-mail: wotanchuang9956162@126.com

*Contributed equally

Key words: microRNA-155, epilepsy, phosphoinositide 3-kinase, protein kinase $\mathrm{B}$, mechanistic target of rapamycin inflexibility, showing paroxysmal feelings, and automatic nervous dysneuria or a combination of these (1). An epidemiological survey showed that the morbidity of epilepsy in general is 50-70 cases/100,000 individuals (2). Following establishment of treatment, $\sim 80 \%$ of epilepsy attacks can be controlled, but $20 \%$ of patients have poor outcomes (3). Epilepsy is a type of chronic or repeatedly paroxysmal disease. Long-term repeated seizures may result in hypophrenia, insanity, a poor memory and reductions in the ability for social adaptation for numerous patients. This may seriously impact the life and work of a patient and their ability to learn (4). If children or adolescents suffer from epilepsy, intelligence and physical development may be more easily impacted. Epilepsy drugs administered for a long time may make certain patients drowsy, tired and inattentive (5). Furthermore, it is hard for epilepsy patients, particularly infants, to achieve a normal education and to be capable of mastering normal work (6). Therefore, epilepsy attacks not only bring pain to the bodies and spirits of patients and their families, but also increases the economic burden of health care. This directly impacts quality of life, particularly in the case of damage to the central nervous system caused by status epilepticus, which is serious with a longer duration. Damage caused to hippocampal neurons is even more serious and impacts the quality of life of patients (7).

MicroRNAs (miRNAs) are small non-coding RNA molecules with a length of 20-24 nt. miRNA are cut by pre-miRNA with a length of 60-70 nt and a hairpin structure (8). In plant and animal cells, miRNA can restrain gene activity or degrade target genes to regulate expressive activity following transcription through interaction with the target gene's specific sequences (9). Every type of miRNA can participate in growth, development, aging and death, as miRNAs have high stability, conservatism, time sequence and tissue specificity in terms of system evolution between species (10).

Among the existing miRNAs, $70 \%$ are expressed in the brain tissues of mammals, including certain miRNAs (miRNA-124a, miRNA-128 and miRNA-101) specific to the brain tissues. Brain tissues are rich in miRNAs (such as miRNA-125b) (11), which express and regulate different physiological processes and conduction pathways in the nervous system, and serve an important role in the initiation and development of the nervous system, neural stem cell differentiation and cell apoptosis (12). It has been shown that the brain miRNA-133 level in patients with Parkinson's disease is markedly reduced compared with 
that in normal control patients. miRNA-25 downregulation in Alzheimer's disease has synergistic effects with ROS (12).

The phosphoinositide 3-kinase (PI3K)/protein kinase B (Akt) signaling pathway serves an important function in the proliferation, differentiation, survival and migration of regulating cells, and is an extremely important conduction pathway of 'survival signals' (it is also known as the 'apoptosis resistance' pathway) (13). PI3K can activate phosphorylated (p-)Akt level to develop its biological functions, and it is an important indicator of standing for the access activity (14). p-Akt can activate multiple downstream proteins, including Bcl-2-associated agonist of cell death (Bad), caspase-3, nuclear factor- $\kappa \mathrm{B}, \mathrm{Kp} 85$, mechanistic target of rapamycin (mTOR), apoptosis regulator $\mathrm{Bcl}-2(\mathrm{Bcl}-2)$ and apoptosis regulator $\mathrm{BAX}$ (Bax), so as to mediate the growth of induced cells. In the signal transduction anti-apoptosis pathway, activation of the PI3K/Akt signal transduction pathway can be regarded as a signal of cell growth and anti-apoptosis (15).

mTORs are highly conservative serine/threonine protein kinases that have multiple activation methods. PI3K/Akt/mTOR signaling pathway is the most common method to be induced by growth factors and superfamily (16). The activated Akt can suspend its inhibitory effects on mTOR, leading to mTOR activation by the inhibition of tuberous sclerosis (TSC)1/TSC2 compound activity relief. In addition to hypoxia, amino acid level and DNA damage, multiple physiological and pathological factors may activate or restrain mTORs (17). mTORs generate a complicated biological effect by forming the functional compounds mTOR complex 1 (mTORC1) and mTORC2 (16). The present study aimed to detect the contribution of miRNA-155 to the occurrence of epilepsy, and to investigate its neuroprotective effect and mechanism.

\section{Materials and methods}

Ethical considerations and patient recruitment. Patients with temporal lobe epilepsy (age, $72 \pm 6.5$ years) and volunteers (age, 68 \pm 5.5 years) were enrolled from the Department of Neurology, Ya'an Hospital (Ya'an, Sichuan, China). The protocol was approved by the Ethics Committee of Ya'an Hospital. The study was conducted in accordance with the guidelines and the principles expressed in Ya'an Hospital.

Reverse transcription-quantitative polymerase chain reaction $(R T-q P C R)$. Blood from patients with temporal lobe epilepsy and volunteers was collected and centrifuged at 3,000 x g for $10 \mathrm{~min}$ at $4^{\circ} \mathrm{C}$. Total RNA was isolated from the serum using TRIzol (Invitrogen; Thermo Fisher Scientific, Inc., Waltham, MA, USA) according to the manufacturer's protocol. Total RNA $(1 \mu \mathrm{g})$ was reverse-transcribed using a RevertAid First Strand cDNA synthesis kit (Thermo Fisher Scientific, Inc.). RT-qPCR was performed using an ABI Mx3000P qPCR system (Stratagene; Agilent Technologies, Inc., Santa Clara, CA, USA) with the Platinum SYBR-Green qPCR Super Mix UDG (Invitrogen; Thermo Fisher Scientific, Inc.) and according to the manufacturer's instructions. The primer sequences for quantitative RT-PCR were as follows: miR-155-5p forward, 5'-GGTCCTTAATGCTAATCGTGATAGGGG-3' and reverse, 5'-CCAGTGCAGGGTCCGAGGT-3'; U6 forward, 5'-TGCGGGTGCTCGCTTCGGCAGC-3' and reverse,

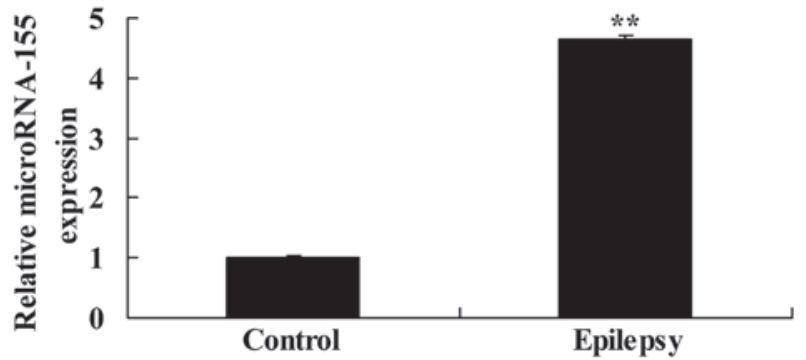

Figure 1. MicroRNA-155 expression in patients with temporal lobe epilepsy. ${ }^{* *} \mathrm{P}<0.01$ compared with control. Control, control group; epilepsy, patients with temporal lobe epilepsy group.

5'-CCAGTGCAGGGTCCGAGGT-3'. miRNA-155 expression level was calculated by the comparative $\mathrm{Cq}$ method (18).

Hippocampal HT22 cell culture and transfection. Mouse hippocampal HT22 cells were purchased from the Shanghai Cell Bank of the Chinese Academy of Sciences (Shanghai, China) and grown in Dulbecco's modified Eagle's medium (Promega Corporation, Madison, WI, USA) containing 10\% (v/v) fetal bovine serum (Gibco; Thermo Fisher Scientific, Inc.) and incubated in a humidified atmosphere $\left(95 \% \mathrm{O}_{2}, 5 \% \mathrm{CO}_{2}\right)$ at $37^{\circ} \mathrm{C}$. The cells were treated with $5 \mathrm{mM}$ glutamate for $24 \mathrm{~h}$. miRNA-155 (5'-UUAAUGCUAAUCGUGAUAGGGGU-3') and negative control plasmid (5'-UUCUCCGAACGUGUCACGUTT-3') were structured and purchased by Sangon Biotech (Shanghai) Co., Ltd. (Shanghai, China). The HT22 cells were then seeded in a 6-well plate and transfected with $100 \mathrm{ng}$ of miRNA-155 or 100 ng negative control plasmid using Lipofectamine ${ }^{\circledR} 2000$. Following transfection for $48 \mathrm{~h}$, the cells were used for experimentation. Following transfection for 24 h, 50 nM LY294002 was added to the HT22 cells for $24 \mathrm{~h}$, which were used for other experiments.

Determination of brain-derived neurotrophic factor (BDNF) and caspase-3 activity by enzyme-linked immunosorbent assay. HT22 cells were lysed in a cold RIPA lysis buffer (Beyotime, Shanghai, China) for $30 \mathrm{~min}$ at $4^{\circ} \mathrm{C}$ and then the supernatant was collected following centrifugation at $2,000 \mathrm{xg}$ for $20 \mathrm{~min}$ at $4^{\circ} \mathrm{C}$. Total protein concentration was determined using the commercial bicinchoninic acid (BCA) assay kit (Beyotime Institute of Biotechnology, Haimen, China). Total proteins $(10 \mu \mathrm{g})$ were incubated with BDNF (H069; Nanjing Jiancheng Biology Engineering Institute, Nanjing, China) and caspase-3 (G015; Nanjing Jiancheng Biology Engineering Institute) activity kits. Optical density was obtained by a microplate reader at a wavelength of 450 or $405 \mathrm{~nm}$.

Western blotting. To evaluate the function of miRNA-155 in epilepsy, western blotting was used to measure the protein expression of BDNF and TrkB protein expression. HT22 cells were lysed in a cold RIPA lysis buffer (Beyotime Institute of Biotechnology) for $30 \mathrm{~min}$ at $4^{\circ} \mathrm{C}$ and then the supernatant was collected following centrifugation at $2,000 \mathrm{x}$ g for $20 \mathrm{~min}$ at $4^{\circ} \mathrm{C}$. Total protein concentration was determined by the commercial BCA assay kit (Beyotime Institute of Biotechnology). Total proteins $(50 \mu \mathrm{g})$ were separated using $6-10 \%$ sodium dodecyl sulfate-polyacrylamide gel electrophoresis and then transferred 

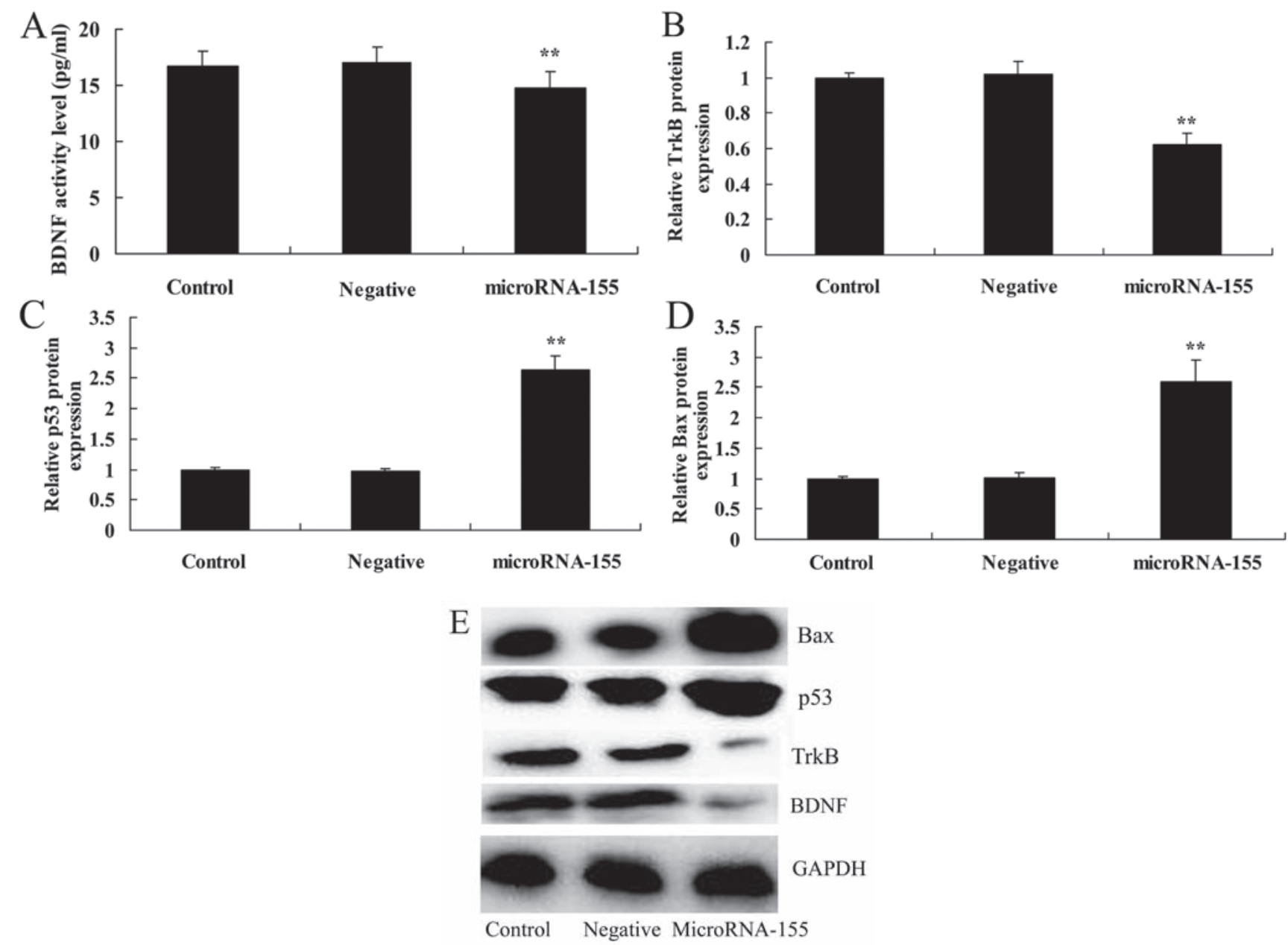

Figure 2. Overexpression of miRNA-155 decreases BDNF and TrkB protein expression and increases p53 and Bax protein expression. Overexpression of miRNA-155 decreased (A) BDNF and TrkB (B) protein expression, and increased (C) p53 and (D) Bax protein expression, as determined by statistical analysis of (E) western blot assays. ${ }^{* *} \mathrm{P}<0.01$ compared with control. Control, control group; negative, negative control group; miRNA-155, overexpression of miRNA-155 group; BDNF, brain-derived neurotrophic factor; TrkB, tropomyosin receptor kinase B; p53, tumor protein p53; Bax, apoptosis regulator BAX; GAPDH, glyceraldehyde 3-phosphate dehydrogenase; miRNA, microRNA.

electrophoretically onto nitrocellulose membranes. Membranes were blocked using 5\% skimmed milk for $1 \mathrm{~h}$ at room temperature and then incubated with anti-BDNF (1:1,000; sc-20981; Santa Cruz Biotechnology, Santa Cruz, CA, USA), anti-TrkB (1:2,000; cat. no. 4607; Cell Signaling Technology,Inc., Danvers, MA, USA) anti-tumor protein p53 (p53; 1:1,000; sc-47698; Santa Cruz Biotechnology), anti-Bax (1:1,000; sc-6236; Santa Cruz Biotechnology), anti-PI3K (1:1,000; sc-1331; Santa Cruz Biotechnology), anti-p-Akt (1:1,000; sc-7985-R; Santa Cruz Biotechnology), anti-p-mTOR (1:1,000; sc-293133; Santa Cruz Biotechnology) and anti-glyceraldehyde 3-phosphate dehydrogenase (GaPDH; 1:5,000; sc-32233; Santa Cruz Biotechnology) overnight at $4^{\circ} \mathrm{C}$. The membranes were washed with TBST and then incubated with the secondary antibodies (1:5,000; sc-2004 or sc-2005; Santa Cruz Biotechnology) for $2 \mathrm{~h}$ at room temperature. Protein visualization was performed using an enhanced chemiluminescence kit and quantified using Quantity One software (both Bio-Rad Laboratories, Inc., Hercules, CA, USA).

Statistical analysis. Data are expressed as the mean \pm standard error. Statistical significance was evaluated using Student's t-test for two groups or one-way analysis of variance with Tukey's post hoc test for multiple comparisons, and was performed using

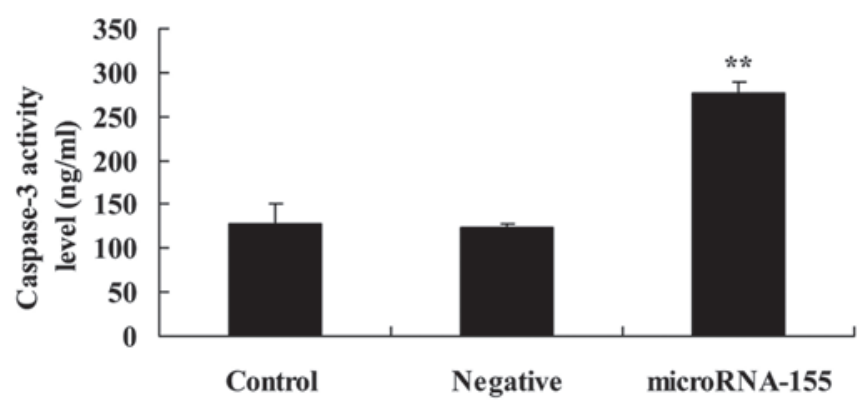

Figure 3. Overexpression of miRNA-155 increases caspase-3 activity. ${ }^{* * *} \mathrm{P}<0.01$ compared with control. Control, control group; negative, negative control group; miRNA-155, overexpression of microRNA-155 group. miRNA, microRNA.

SPSS 13.0 software (SPSS, Inc., Chicago, IL, USA). P<0.05 was considered to indicate a statistically significant difference.

\section{Results}

miRNA-155 expression in patients with temporal lobe epilepsy. miRNA-155 expression was selected for assessment in patients with temporal lobe epilepsy and volunteers in the present 

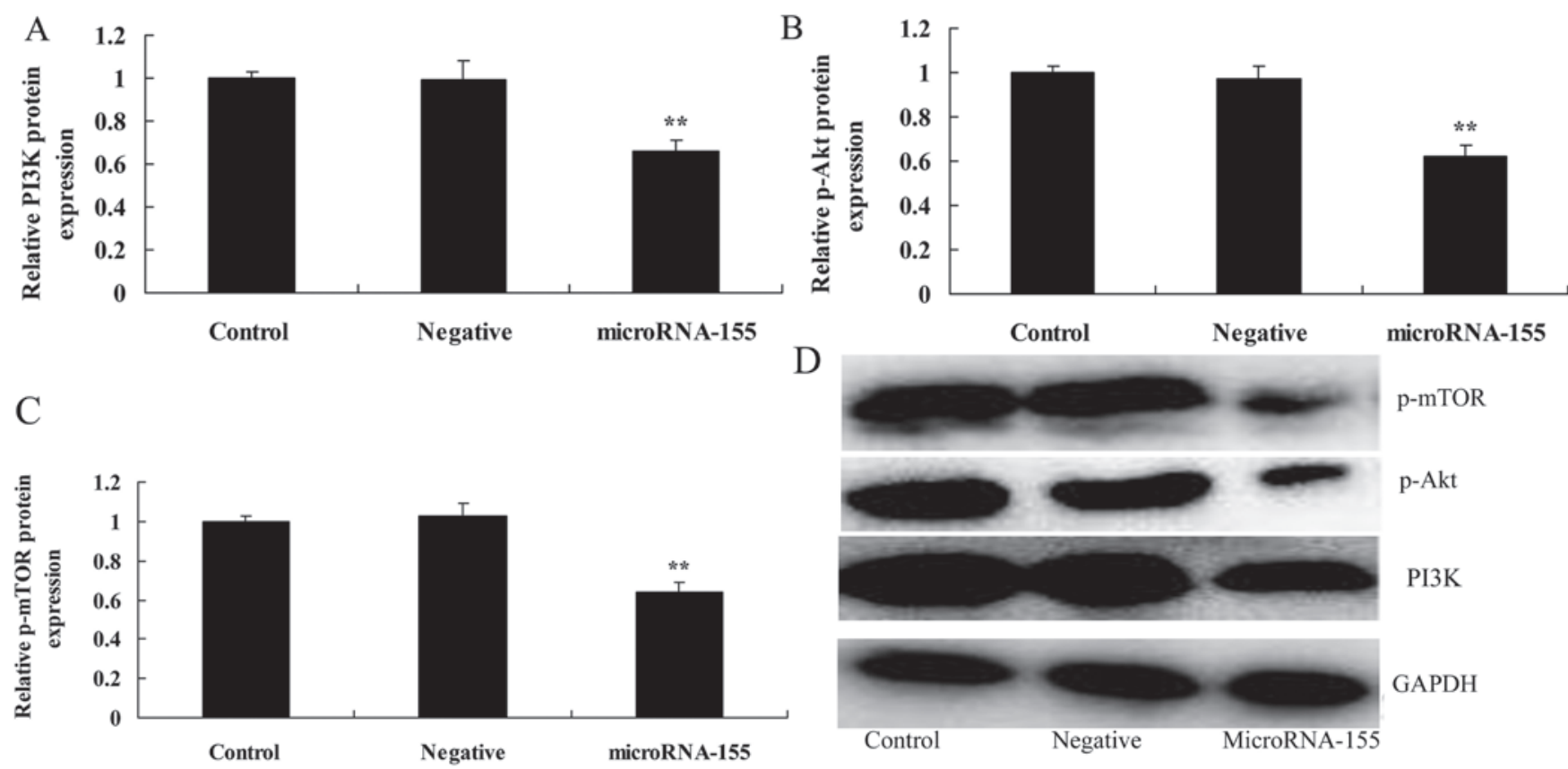

Figure 4. Overexpression of miRNA-155 inhibits the PI3K/Akt/mTOR signaling pathway. Overexpression of miRNA-155 inhibited (A) PI3K, (B) p-Akt and (C) p-mTOR protein expression, as determined by statistical analysis and (D) western blot assays. ${ }^{* *} \mathrm{P}<0.01$ compared with control. Control, control group; negative, negative control group; miRNA-155, overexpression of miRNA-155 group; PI3K, phosphoinositide 3-kinase; Akt, protein kinase B; mTOR, mechanistic target of rapamycin; p-, phosphorylated; GAPDH, glyceraldehyde 3-phosphate dehydrogenase; miRNA, microRNA.

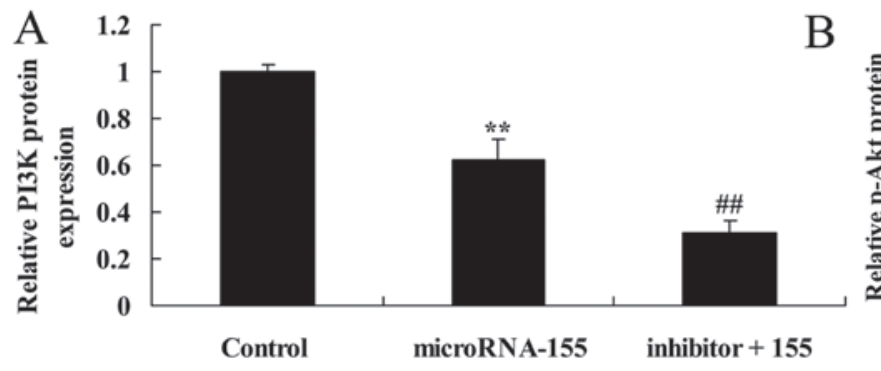

C

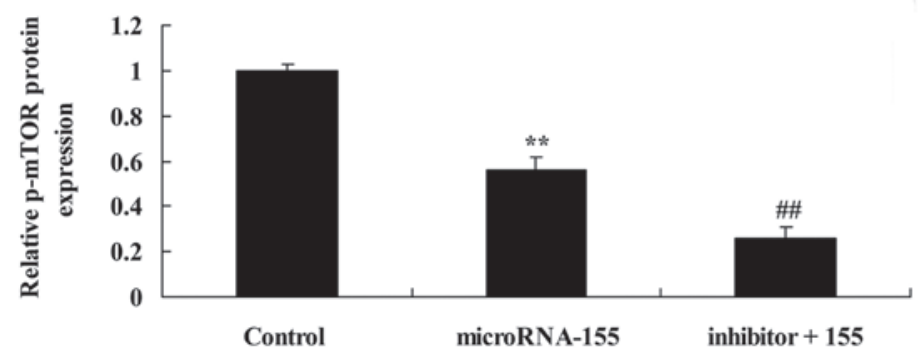

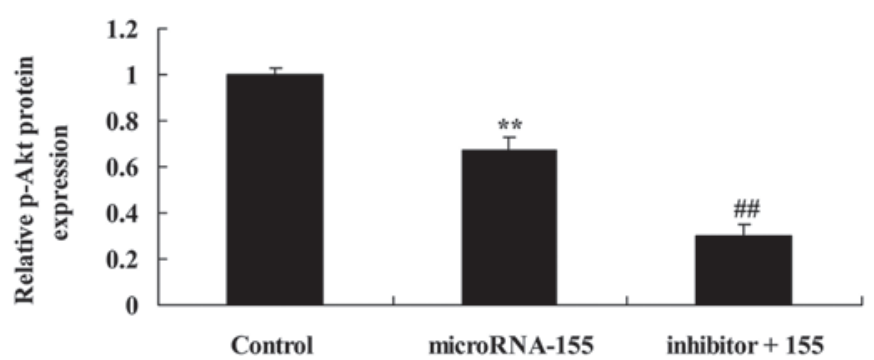

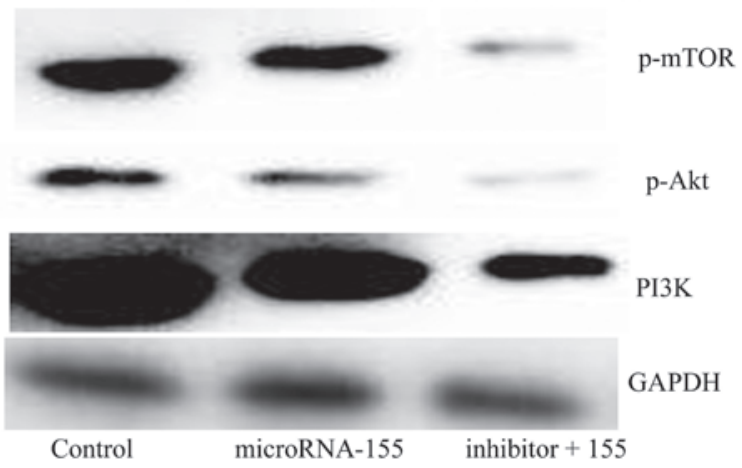

Figure 5. Inhibition of PI3K accelerates the effect of miRNA-155 on the suppression of the PI3K/Akt/mTOR signaling pathway. Inhibition of PI3K accelerated the effect of miRNA-155 on the inhibition of (A) PI3K, (B) p-Akt and (C) p-mTOR protein expression, as determined by statistical analysis and (D) western blot assays. ${ }^{* *} \mathrm{P}<0.01$ compared with control group; ${ }^{\# /} \mathrm{P}<0.01$ compared with miRNA-155 group. Control, control group; miRNA-155, overexpression of miRNA-155 group; inhibitor + 115, PI3K inhibitor + overexpression of miRNA-155 group; PI3K, phosphoinositide 3-kinase; Akt, protein kinase B; mTOR, mechanistic target of rapamycin; p-, phosphorylated; GAPDH, glyceraldehyde 3-phosphate dehydrogenase; miRNA, microRNA.

study. The miRNA-155 expression in patients with temporal lobe epilepsy was significantly higher than that of the control volunteer group (Fig. 1).

Overexpression of miRNA-155 decreases BDNF and tropomyosin receptor kinase $B$ (TrkB) protein expression. To evaluate the overexpression of miRNA-155 in epilepsy, BDNF and TrkB protein expression in HT22 cells under glutamate stimulation was measured (Fig. 2). As displayed in Fig. 2A, B and E, overexpression of miRNA-155 decreased BDNF and TrkB protein expression in HT22 cells under glutamate stimulation compared with that in the control or negative groups. 
A
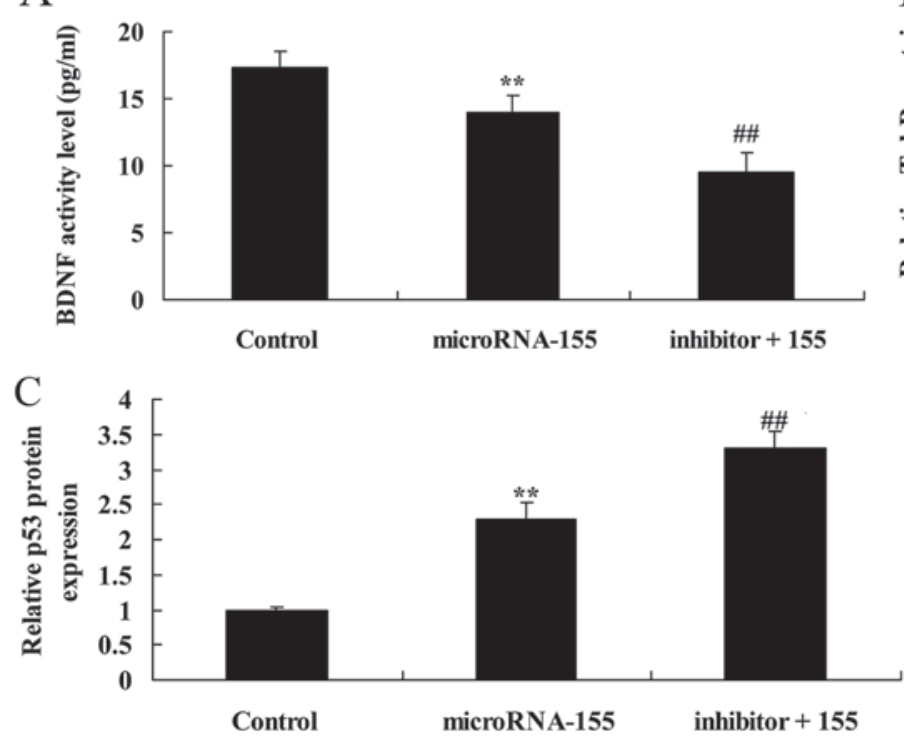
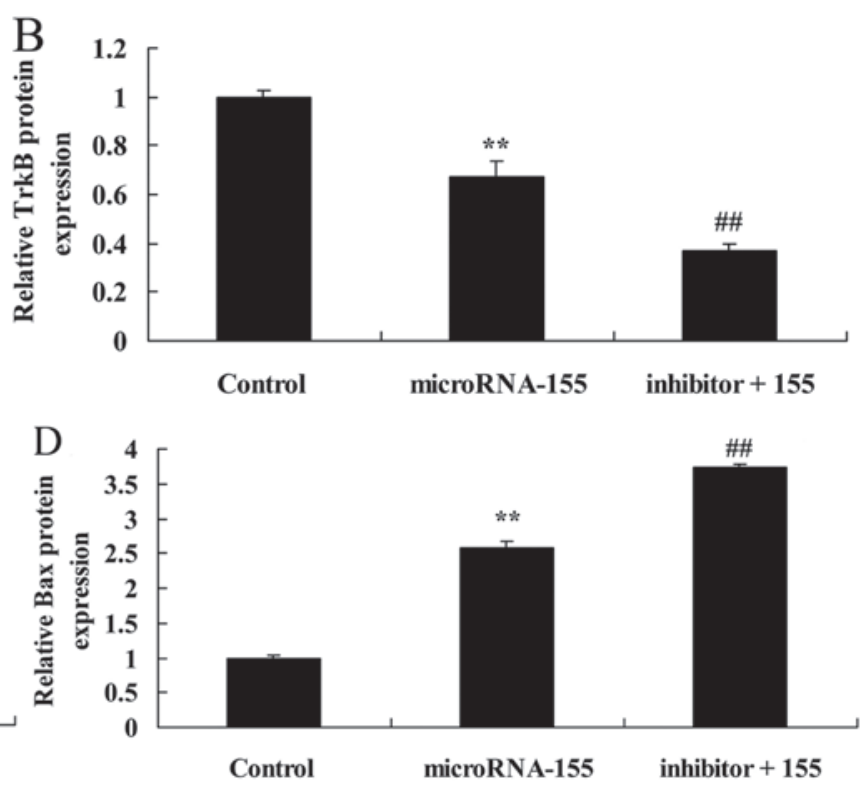

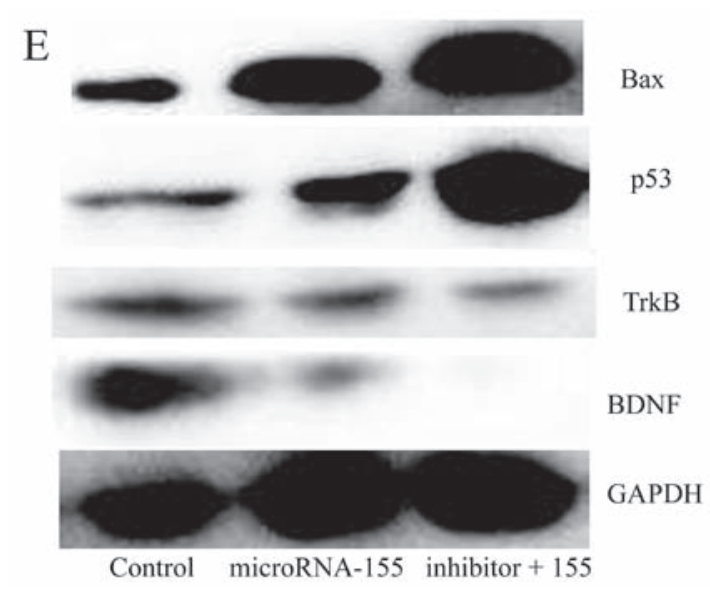

Figure 6. Inhibition of PI3K accelerates the effect of miRNA-155 inhibition of BDNF and TrkB protein expression, and induction of p53 and Bax protein expression. Inhibition of PI3K accelerated the effect of miRNA-155 inhibition of (A) BDNF, (B) TrkB, (C) p53 and (D) Bax protein expression, as determined by statistical analysis and (E) western blot assays. ${ }^{* *} \mathrm{P}<0.01$ compared with control; ${ }^{\# \#} \mathrm{P}<0.01$ compared with miRNA-155. Control, control group; miRNA-155, overexpression of miRNA-155 group; inhibitor + 115, PI3K inhibitor + overexpression of miRNA-155 group; PI3K, phosphoinositide 3-kinase; BDNF, brain-derived neurotrophic factor; TrkB, tropomyosin receptor kinase B; miRNA, microRNA; p53, tumor protein p53; Bax, apoptosis regulator BAX; GAPDH, glyceraldehyde 3-phosphate dehydrogenase.

Overexpression of miRNA-155 increases caspase-3 activity. To investigate whether the overexpression of miRNA-155 induced apoptosis of epilepsy, caspase-3 activity in HT22 cells under glutamate stimulation was measured in the present study. As shown in Fig. 3, overexpression of miRNA-155 increased caspase-3 activity in the HT22 cells under glutamate stimulation compared with that in the negative control group.

Overexpression of miRNA-155 increases 553 protein expression. The present study further investigated whether the overexpression of miRNA-155 induced apoptosis of epilepsy by measuring p53 protein expression. Fig. $2 \mathrm{C}$ and $\mathrm{E}$ shows that the overexpression of miRNA-155 could increase the p53 protein expression in HT22 cells under glutamate stimulation compared with that in the negative control group.

Overexpression of miRNA-155 induces Bax protein expression. Next, the present study investigated whether the overexpression of miRNA-155 induced Bax protein expression in epilepsy.
As shown in Fig. 2D and E, overexpression of miRNA-155 induced Bax protein expression in HT22 cells under glutamate stimulation compared with that in the negative control group.

Overexpression of miRNA-155 inhibits the PI3K/Akt signaling pathway. miRNA-155 in the PI3K/Akt signaling pathway was also investigated in this study (Fig. 4). Fig. 4A, B and D shows that the overexpression of miRNA-155 suppressed the PI3K/Akt signaling pathway, and inhibited PI3K and p-Akt protein expression in HT22 cells under glutamate stimulation compared with that in the negative control group.

Overexpression of miRNA-155 inhibits mTOR protein expression. Downstream of PI3K/Akt signaling is mTOR, which was investigated to enrich our analysis of miRNA-155 in epilepsy. The overexpression of miRNA-155 also inhibited the mTOR signaling pathway, and suppressed p-mTOR protein expression in HT22 cells under glutamate stimulation compared with that in the negative control group (Fig. 4C and D). 


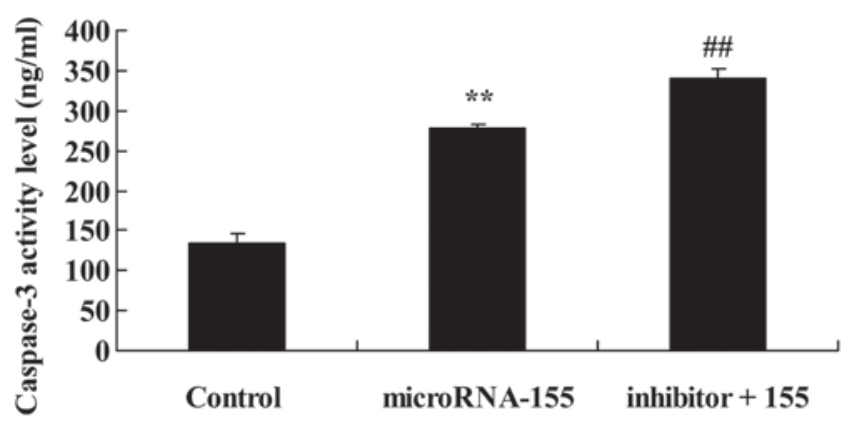

Figure 7. Inhibition of PI3K accelerates the effect of miRNA-155 on the induction of caspase-3 activity. Control, control group; miRNA-155, overexpression of miRNA-155 group; inhibitor +115 , PI3K inhibitor + overexpression of miRNA-155 group; PI3K, phosphoinositide 3-kinase; miRNA, microRNA.

Inhibition of PI3K accelerates the effect of miRNA-155 on the suppression of the PI3K/Akt/mTOR signaling pathway. According to the aforementioned results, the PI3K/Akt signaling pathway may participate in the effect of miRNA-155 on epilepsy. PI3K inhibitor suppressed the PI3K/Akt signaling pathway and inhibited PI3K, p-Akt and p-mTOR protein expression in HT22 cells under glutamate stimulation following miRNA-155 overexpression (Fig. 5).

Inhibition of PI3K accelerates the effect of miRNA-155 on the suppresion BDNF and TrkB protein expression. The change in BDNF and TrkB protein expression was investigated whilst assessing the effect of miRNA-155 on epilepsy (Fig. 6). Inhibition of PI3K decreased the BDNF and TrkB protein expression in HT22 cells under glutamate stimulation following miRNA-155 overexpression (Fig. 6A, B and E).

Inhibition of PI3K accelerates the effect of miRNA-155 on the induction of caspase-3 activity. The PI3K/Akt signaling pathway is concerned with the apoptosis mechanism of miRNA-155 in epilepsy. As shown in Fig. 7, inhibition of PI3K increased caspase-3 activity in HT22 cells under glutamate stimulation following miRNA-155 overexpression.

Inhibition of PI3K accelerates the effect of miRNA-155 on the induction of p53 and Bax protein expression. The PI3K/Akt signaling pathway serves a critical role in miRNA-155 in epilepsy. As shown in Fig. 6C-E, inhibition of PI3K promoted p53 and Bax protein expression in HT22 cells under glutamate stimulation following miRNA-155 overexpression.

\section{Discussion}

Epilepsy is a brain disease with features that generate a susceptibility to continuous epileptic attack and that also present with corresponding neurobiological, cognitive, psychological and societal consequences (19). Epilepsy is a common disease of the nervous system that seriously threatens human health, not only doing harm to the patients, but also conferring a heavy burden on the family and on society. A domestic epidemiological survey showed that the prevalence rate of epilepsy is $0.7 \%$ (2). Thus, it is predicted that there are $\sim 9$ million patients globally, with $1 / 3$ patients experiencing refractory epilepsy. The definition of refractory epilepsy has not been reached by consensus thus far (3). The majority of scholars suggest that sequential or combined applications of 3 or more anti-epilepsy drugs can provide a sufficient or tolerated dosage, and have observed a sufficiently long course of treatment (20). In refractory epilepsy, the attack times do not reduce or even increase slightly as a result (21). The findings of the present study indicated that the miRNA-155 expression in patients with temporal lobe epilepsy was significantly higher than that of the control volunteer group.

As it is hard to extract materials from the human brain of epilepsy patients, it is also difficult to directly detect the miRNA expression in human brain tissues in vitro (10). Cerebrospinal fluid is mainly generated in the choroid plexus tissues, and is created and absorbed into the veins constantly (22). The fluid serves a lymphatic role in the central nervous system; it provides a certain degree of nutrition to the brain cells, carries away metabolites of brain tissues, regulates the acid-base balance of the central nervous system, reduces the pressure in the brain and spinal cord, and protects and supports the brain and spinal cord (23). Meanwhile, cerebrospinal fluid envelops the brain parenchyma, can contact the external cell gap directly, and can reflect the pathological and physiological changes of brain tissues dynamically (22). Soluble molecular biomarkers in cerebrospinal fluid are of value in studying brain diseases (24). miRNAs in the cerebrospinal fluid can be regarded as potential biomarkers in the central nervous system, particularly for Alzheimer's disease, Huntington's chorea, disseminated sclerosis, schizophrenia and bipolar affective disorder (25). Furthermore, the present data also evidently showed that overexpression of miRNA-155 decreased BDNF level and TrkB protein expression in HT22 cells under glutamate stimulation.

In the process of an epileptic attack, repeated seizures may cause ischemia and anoxia of brain tissues, release excitatory amino acids and cause an inward sodium current, so as to launch a caspase chain reaction (26). Epilepsy can generate free radicals, nerve-nitric oxide synthase, mediate apoptosis core and execute protein kinase caspase-3, make important protein degraded and inactivated, such as cytoplasm, cell nucleus and cytoskeleton and result in the apoptosis and deficiency of numerous nerve cells (27). Nerve cell damage is one of the reasons for chronic spontaneous epilepsy (28). Finally it may form refractory TLE. The morbidity of TLE accounts for $25 \%$ of epilepsy cases. In refractory epilepsy, even once patients have been treated using multiple anti-epilepsy drugs, the attacks still cannot be controlled (28). Therefore, controlling the deficiency of nerve cells after an epileptic attack has important significance for refractory epilepsy. Epilepsy nerve cell apoptosis is regulated by a series of genes, including miRNAs, Bcl-2, Bax and p53 (29). The abnormal expression of these genes serves an important role in the apoptosis of nerve cells. The apoptosis of the nerve cells occurs by launching the internal death mechanism of the cells. Studies on relevant genes associated with epilepsy nerve apoptosis are increasing in number. In the present study, overexpression of miRNA-155 increased caspase-3 activity, and p53 and Bax expression, and reduced PI3K, p-Akt and p-mTOR protein expression in epilepsy cells.

The PI3K/Akt signal transduction pathway is widely applied in cells. PI3K is the dimer protein with a P110 catalytic subunit 
and a p85 regulating subunit. PI3K has protein kinases and lipoid kinase activity (15). Akt is the direct downstream substrate of $\mathrm{PI} 3 \mathrm{~K}$ with a relative molecular mass is $60 \mathrm{kDa}$. Akt serves an important role in the signal transduction pathway. PI3K and JAK2 activation induced erythropoeitin (EPO), which caused p-Akt protein expression (30). p-JAK2 makes PI3K regulated subunits to combine. When the regulated subunit is combined with EPO-R, the catalytic subunit is activated and phosphorylates Akt. p-Akt induced $\mathrm{Bcl}-2$ protein expression and suppressed caspase- 9 protein expression. In summary, activated Akt can phosphorylate apoptosis proteins or change the expression level of apoptosis genes indirectly, so as to regulate the apoptotic process (31). The specific mechanism refers to restraining the activation of caspase family members and restraining the apoptosis caused by caspase; releasing apoptosis by reducing release of $\mathrm{CytC}$; regulating activity of Bcl-2 family members, making Bad and Bax residues phosphorylated and making them inactivated and restraining apoptosis (27). The present study showed that PI3K inhibitor accelerated the effect of miRNA-155 on the inhibition of BDNF and TrkB protein expression, and on the promotion of caspase-3 activity and p53 and Bax protein expression in epilepsy cells.

According to existing research results, the mTOR signaling pathway participates in multiple of pathological changes at the modular and cellular level, including apoptosis, gliosis, and changes to synaptic plasticity, neurotransmitter receptor, ion channel and axon budding, associated with epilepsy (32). In genetic diseases, including TSC, PMSE, sporadic diseases PCD and GG, and acquired epilepsy, the mTOR signal pathway is activated abnormally (32). Sirolimus affects the mTOR signaling pathway; it can reduce an epilepsy attack to a certain extent or reverse pathological changes (33). Taken together, the results of the present study indicated that the inhibition of PI3K enhanced the effect of miRNA-155 on p-mTOR protein expression in epilepsy cells.

In conclusion, the present study demonstrated that miRNA-155 contributes to the occurrence of epilepsy, and also provided evidence that miRNA-155 exhibits a neuroprotective effect on epilepsy-induced neuronal apoptosis through the $\mathrm{PI} 3 \mathrm{~K} / \mathrm{Akt} / \mathrm{mTOR}$ signaling pathway. These findings suggest that miRNA-155 induced neuronal apoptosis in epilepsy, which may open up new avenues in the treatment of refractory epilepsy.

\section{Acknowledgements}

Not applicable.

\section{Funding}

No funding was received.

\section{Availability of data and materials}

All data generated or analyzed during this study are included in this published article.

\section{Authors' contributions}

XRW designed the experiment. WD and YC performed the experiment and analyzed the data. XRW wrote the manuscript. All authors read and approved the final manuscript.

\section{Ethics approval and consent to participate}

The protocol was approved by the Ethics Committee of Ya'an Hospital. The study was conducted in accordance with the guidelines and the principles expressed in Ya'an Hospital.

\section{Patient consent for publication}

Not applicable.

\section{Competing interests}

The authors declare that they have no competing interests.

\section{References}

1. Lazzari AA, Dussault PM, Thakore-James M, Gagnon D, Baker E, Davis SA and Houranieh AM: Prevention of bone loss and vertebral fractures in patients with chronic epilepsy - antiepileptic drug and osteoporosis prevention trial. Epilepsia 54: 1997-2004, 2013.

2. Delger AB, Avakyan GN, Oleinikova OM, Bogomazova MA, Chromych EA and Lagutin IuV: Effects of tenoten on anxiety and depression disorders in patients with epilepsy. Bull Exp Biol Med 153: 704-706, 2012.

3. Jacoby A, Sudell M, Tudur Smith C, Crossley J, Marson AG and Baker GA; SANAD Study Group: Quality-of-life outcomes of initiating treatment with standard and newer antiepileptic drugs in adults with new-onset epilepsy: Findings from the SANAD trial. Epilepsia 56: 460-472, 2015.

4. Jóźwiak S, Kotulska K, Domańska-Pakieła D, Lojszczyk B, Syczewska M, Chmielewski D, Dunin-Wąsowicz D, Kmieć T, Szymkiewicz-Dangel J, Kornacka M, et al: Antiepileptic treatment before the onset of seizures reduces epilepsy severity and risk of mental retardation in infants with tuberous sclerosis complex. Eur J Paediatr Neurol 15: 424-431, 2011.

5. French JA, Abou-Khalil BW, Leroy RF, Yacubian EM, Shin P, Hall S, Mansbach H and Nohria V; RESTORE 1/Study 301 Investigators: Randomized, double-blind, placebo-controlled trial of ezogabine (retigabine) in partial epilepsy. Neurology 76: 1555-1563, 2011.

6. Sharma S, Sankhyan N, Gulati S and Agarwala A: Use of the modified Atkins diet for treatment of refractory childhood epilepsy: A randomized controlled trial. Epilepsia 54: 481-486, 2013.

7. Kerr EN and Blackwell MC: Near-transfer effects following working memory intervention (Cogmed) in children with symptomatic epilepsy: An open randomized clinical trial. Epilepsia 56: 1784-1792, 2015.

8. Li MM, Li XM, Zheng XP, Yu JT and Tan L: MicroRNAs dysregulation in epilepsy. Brain Res 1584: 94-104, 2014.

9. Ashhab MU, Omran A, Kong H, Gan N, He F, Peng J and Yin F: Expressions of tumor necrosis factor alpha and microRNA-155 in immature rat model of status epilepticus and children with mesial temporal lobe epilepsy. J Mol Neurosci 51: 950-958, 2013.

10. Li Y, Wang J, Jiang C, Zheng G, Lu X and Guo H: Association of the genetic polymorphisms in pre-microRNAs with risk of childhood epilepsy in a Chinese population. Seizure 40: 21-26, 2016.

11. Li MM, Jiang T, Sun Z, Zhang Q, Tan CC, Yu JT and Tan L: Genome-wide microRNA expression profiles in hippocampus of rats with chronic temporal lobe epilepsy. Sci Rep 4: 4734, 2014.

12. Henshall DC: MicroRNA and epilepsy: Profiling, functions and potential clinical applications. Curr Opin Neurol 27: 199-205, 2014.

13. Xue Y, Xie N, Cao L, Zhao X, Jiang H and Chi Z: Diazoxide preconditioning against seizure-induced oxidative injury is via the PI3K/Akt pathway in epileptic rat. Neurosci Lett 495: 130-134, 2011.

14. Xiao Z, Peng J, Yang L, Kong H and Yin F: Interleukin-1 $\beta$ plays a role in the pathogenesis of mesial temporal lobe epilepsy through the PI3K/Akt/mTOR signaling pathway in hippocampal neurons. J Neuroimmunol 282: 110-117, 2015. 
15. Zheng H, Wang X, Tang Z, Zheng W and Li Z: The PI3K/ Akt and ERK1/2 signaling pathways mediate the erythropoietin-modulated calcium influx in kainic acid-induced epilepsy. Neuroreport 24: 335-341, 2013.

16. Wong M: mTOR strikes again: mTORC1 activation causes epilepsy independent of overt pathological changes. Epilepsy Curr 14: 41-43, 2014.

17. Bockaert J and Marin P: mTOR in brain physiology and pathologies. Physiol Rev 95: 1157-1187, 2015.

18. Livak KJ and Schmittgen TD: Analysis of relative gene expression data using real-time quantitative PCR and the 2(-Delta Delta C(T)) Method. Methods 25: 402-408, 2001.

19. Aalbers MW, Klinkenberg S, Rijkers K, Verschuure P, Kessels A, Aldenkamp A, Vles J and Majoie M: The effects of vagus nerve stimulation on pro- and anti-inflammatory cytokines in children with refractory epilepsy: An exploratory study. Neuroimmunomodulation 19: 352-358, 2012.

20. Meurer WJ, Silbergleit R, Nicholas KS, Burke JF and Durkalski V: Accounting for repeat enrollments during an emergency clinical trial: The Rapid Anticonvulsant Medications Prior to Arrival Trial (RAMPART). Acad Emerg Med 22: 373-377, 2015.

21. Ryvlin P, Gilliam FG, Nguyen DK, Colicchio G, Iudice A Tinuper P, Zamponi N, Aguglia U, Wagner L, Minotti L, et al: The long-term effect of vagus nerve stimulation on quality of life in patients with pharmacoresistant focal epilepsy: The PuLsE (Open Prospective Randomized Long-term Effectiveness) trial. Epilepsia 55: 893-900, 2014

22. Hu K, Xie YY, Zhang C, Ouyang DS, Long HY, Sun DN, Long LL, Feng L, Li Y and Xiao B: MicroRNA expression profile of the hippocampus in a rat model of temporal lobe epilepsy and miR-34a-targeted neuroprotection against hippocampal neurone cell apoptosis post-status epilepticus. BMC Neurosci 13: 115, 2012.

23. Zucchini S, Marucci G, Paradiso B, Lanza G, Roncon P Cifelli P, Ferracin M, Giulioni M, Michelucci R, Rubboli G, et al: Identification of miRNAs differentially expressed in human epilepsy with or without granule cell pathology. PLoS One 9: e105521, 2014

24. Reschke CR and Henshall DC: microRNA and Epilepsy. Adv Exp Med Biol 888: 41-70, 2015.
25. Manna I, Labate A, Borzì G, Mumoli L, Cavalli SM, Sturniolo M, Quattrone A and Gambardella A: An SNP site in pri-miR-124, a brain expressed miRNA gene, no contribution to mesial temporal lobe epilepsy in an Italian sample. Neurol Sci 37: 1335-1339, 2016.

26. Tzeng TT, Tsay HJ, Chang L, Hsu CL, Lai TH, Huang FL and Shiao YJ: Caspase 3 involves in neuroplasticity, microglial activation and neurogenesis in the mice hippocampus after intracerebral injection of kainic acid. J Biomed Sci 20: 90 2013.

27. Aanandhi MV, Bhattacherjee D, Ray A and George PS: New avenue in the treatment of temporal lobe epilepsy by classical anti-epileptics: A hypothetical establishment of executioner Caspase 3 inactivation by molecular modeling. J Adv Pharm Technol Res 6: 65-74, 2015.

28. Meng XJ, Wang F and Li CK: Resveratrol is neuroprotective and improves cognition in pentylenetetrazole-kindling model of epilepsy in rats. Indian J Pharm Sci 76: 125-131, 2014.

29. Chen X, Bao G, Hua Y, Li Y, Wang Z and Zhang X: The effects of topiramate on caspase-3 expression in hippocampus of basolateral amygdala (BLA) electrical kindled epilepsy rat. J Mol Neurosci 38: 201-206, 2009.

30. Zhang B and Wong M: Pentylenetetrazole-induced seizures cause acute, but not chronic, mTOR pathway activation in rat. Epilepsia 53: 506-511, 2012.

31. Jansen LA, Mirzaa GM, Ishak GE, O'Roak BJ, Hiatt JB, Roden WH, Gunter SA, Christian SL, Collins S, Adams C, et al: $\mathrm{PI} 3 \mathrm{~K} / \mathrm{AKT}$ pathway mutations cause a spectrum of brain malformations from megalencephaly to focal cortical dysplasia. Brain 138: 1613-1628, 2015.

32. Avet-Rochex A, Carvajal N, Christoforou CP, Yeung K, Maierbrugger KT, Hobbs C, Lalli G, Cagin U, Plachot C, $\mathrm{McNeill} \mathrm{H}$, et al: Unkempt is negatively regulated by mTOR and uncouples neuronal differentiation from growth control. PLoS Genet 10: e1004624, 2014.

33. Ye M, Bi YF, Ding L, Zhu WW and Gao W: Saikosaponin a functions as anti-epileptic effect in pentylenetetrazol induced rats through inhibiting mTOR signaling pathway. Biomed Pharmacother 81: 281-287, 2016. 